\title{
PATTERNS OF BREAST CANCER RELAPSE AT UNIVERSITY OF MALAYA MEDICAL CENTRE
}

\author{
Rozita AM', Marniza S', Mastura MY', Wan Zamaniah WI', Yip $\mathrm{CH}^{2}$, Taib NA ${ }^{2}$ \\ 1 Clinical Oncology Unit, Faculty of Medicine, University of Malaya, Kuala Lumpur, Malaysia. \\ 2 Department of Surgery, Faculty of Medicine, University of Malaya, Kuala Lumpur, Malaysia.
}

\begin{abstract}
:
Despite being the major cause of cancer-related death in Malaysian women, local data on patterns of breast cancer relapse and their long term outcomes are still scarce. We conducted a retrospective study on all patients treated for non-metastatic invasive breast cancer in 19992000 at the University of Malaya Medical Centre (UMMC), who subsequently developed relapse. We sought to analyse the patterns of relapse, their associated clinicopathological features and the overall survival ratefollowing the relapses. Univariate and multivariate analyses were used to analyse demographics and clinicopathological factors. Survival was analysed using the Kaplan and Meier method and compared by the log rank test. A total of 268 patients with a mean age of 50, were identified for the study. At a median follow-up of 50 months, 73 patients (27.2\%) had relapsed. Local, regional and distant relapse rates were $5.5 \%, 1.9 \%$ and $19.8 \%$ respectively, whereas, the 5 -year survival rates were $61 \%, 40 \%$ and $21 \%$ respectively $(p<0.01$ ). Most relapses occurred within the first five years of diagnosis. Patients with long disease-free interval had better survival. The most common distant relapse site was the lungs while bone was the distant relapse site with the best prognosis. Disease stage, nodal status and oestrogen receptor status were found to have correlation with the risk of relapse. We concluded that the survival of patients with relapsed breast cancer was associated with the site(s) of first relapse and the disease free interval and clinicopathological factors can be used to predict the risk of relapse. (JUMMEC 2010; 13 (1): 24-32)
\end{abstract}

KEYWORDS: relapsed breast cancer, relapse site, survival

\section{Introduction}

Breast cancer is the most common cancer affecting women in Malaysia. The third issue of the National Cancer Registry on cancer incidence in Peninsular Malaysia reported 11,952 new cases of breast cancer in 2003-2005, accounting for $31.3 \%$ of newly diagnosed cancers in women. Breast cancer was also the most common cancer in all ethnic and age groups of females above 15 . The peak age-specific incidence rate was in the $50-60$ years age-group (1).

Breast cancer is also the most frequent cancer in women worldwide. It accounts for $23 \%$ of all cancers. With an estimated number of 1.15 million new cases in 2002, it ranked second overall when both sexes were considered together (2). Despite the increasing trend in the incidence of breast cancer worldwide, survival has steadily improved over the recent decades. This may be explained by the development of improved treatment modalities and earlier detection as a result of effective screening programs and increased awareness among women. Nevertherless, breast cancer remains as the leading cause of death in women worldwide (3).

The aim of treating of non-metastatic invasive breast cancer is to cure. Management of breast cancer in UMMC was evidence-based and later guided by the
Correspondence:
Rozita Abdul Malik
Clinical Oncology Unit
Faculty of Medicine, University of Malaya
50603 Kuala Lumpur, Malaysia
Email: rozita_abdulmalik@yahoo.com 
Ministry of Health Clinical Practice Guidelines on 'Management of Breast Cancer' which was issued in December 2002 (4). The treatment involves a multidisciplinary team which includes breast surgeons, clinical oncologists, pathologists, radiologists and other allied health personnel.

Non-metastatic breast cancer has the tendency to relapse despite adequate curative therapy. The site(s) and the timing of relapse are important determinants of outcome in relapsed breast cancer. They also play a significant role in determining the appropriate management of the patients. Local and/or regional relapse may still be suitable for curative local treatment, whereas patients with distant relapse are usually offered systemic therapy, with palliative intent. The risk of relapse may be estimated based on the clinical and pathological risk factors. Due to this risk, patients are given long term follow-up after completing all curative treatment.

In 2006, Elder et al. reported the results of a study on the patterns of breast cancer relapse of 2509 patients treated at Strathfield Breast Centre (TSBC) in Australia (3). They found that most relapses occurred within the first five years of diagnosis, with the greatest risk between one to two years from primary surgery. The relapse rate was $18 \%$ and bone was the most common site of relapse. The prognosis was dependent on the timing and site(s) of relapse. Late relapses resulted in better survival compared to early relapses. They reported a 5-year survival rate of $41 \%, 20 \%$ and $13 \%$ for local, regional and distant relapse respectively. In patients with distant relapse, the survival rate after relapse in the bones was higher than in the viscera. Two other studies by Imkampe et al. and Giordano et al. also showed similar outcomes based on the sites of relapse $(5,6)$.

Furthermore, these studies also showed the important role of clinicopathological factors in determining breast cancer outcome. Higher stage, large tumour size, positive node and high tumour grade are poor prognostic factors associated with a higher risk of relapse $(3,5,6)$.

In 2008, Taib et al. reported the survival outcome of 413 Malaysian women with breast cancer diagnosed between 1993 and 1997 at the UMMC. The objectives were to analyse the overall survival and the prognostic factors that affect survival. They suggested that ethnicity may be one of the significant prognostic factors in addition to stage, size of tumour, nodal status and tumour grade in influencing survival (7). Oestrogen receptor (ER) status was not found to be a significant prognostic factor in this study.

A study conducted at the University of Texas involving 647 patients showed that ER status had a significant effect on the rate and likely sites of relapse (8). ER negative had a significantly higher rate of relapse compared to ER positive. There was a significantly higher rate of relapse in the viscera and soft tissues in ER negative patients whereas ER positive status was associated with a higher rate of relapse in the bones.

Currently, there is no available published data on the patterns of breast cancer relapse in Malaysia. Therefore, this study was performed to analyse the patterns of breast cancer relapse and the prognosis following relapse.

\section{Methodology}

This was a retrospective study conducted on all women diagnosed with non-metastatic invasive breast cancer in UMMC between 1st January 1999 and 31st December 2000. Patients who defaulted on surgery or adjuvant treatment were excluded from the study. Those who developed relapse were selected for further analysis.

Data on patients' demography, clinicopathological factors, treatment and relapses were obtained from available records. Survival data was obtained from the National Registry of Birth and Death. Patients who did not come for followup were contacted to determine their current status. Uncontactable patients were deemed as 'unknown'. Staging of cancer was based on the 2002 Tumour Node Metastasis (TNM) classification of Malignant-Tumours by the American Joint Committee on Cancer (AJCC).

Site of relapse was categorized into local, regional or distant. Local relapse is occurrence of relapse either in the conserved breast or the chest wall of patients who had undergone mastectomy. 
Regional relapse is disease recurring in the regional lymph nodes either in the ipsilateral axillary, supraclavicular or infraclavicular fossa. Distant or systemic relapse refers to distant metastases. Relapses were recorded according to the first or most significant site of relapse. Simultaneous local and regional disease is categorized as regional relapse while any simultaneous occurrence of distant relapse with local or regional disease was categorized as distant relapse.

Disease free interval (DFI) was defined as the time from the date of primary breast cancer surgery to the date of relapse. Overall survival was defined

Table 1: Patient Demographics

\begin{tabular}{|c|c|c|}
\hline Variable & $n=268$ & $\%$ \\
\hline \multicolumn{3}{|l|}{ Age } \\
\hline$<40$ & 27 & 10.1 \\
\hline $40-49$ & 127 & 58.6 \\
\hline $50-59$ & 62 & 23.1 \\
\hline$\geq 60$ & 22 & 8.2 \\
\hline \multicolumn{3}{|l|}{ Ethnic group } \\
\hline Malay & 53 & 19.8 \\
\hline Chinese & 174 & 64.9 \\
\hline Indian & 37 & 13.8 \\
\hline Others & 4 & 1.5 \\
\hline \multicolumn{3}{|l|}{ Tumour size } \\
\hline$\leq 2 \mathrm{~cm}$ & 95 & 35.4 \\
\hline$>2$ and $\leq 5 \mathrm{~cm}$ & 139 & 51.9 \\
\hline$>5 \mathrm{~cm}$ & 33 & 12.3 \\
\hline Unknown & 1 & 0.4 \\
\hline \multicolumn{3}{|c|}{ Axillary nodes status } \\
\hline Positive 1-3 & 82 & 30.6 \\
\hline Positive 4-9 & 36 & 13.4 \\
\hline Positive $>10$ & 16 & 6.0 \\
\hline Negative & 134 & 50.0 \\
\hline \multicolumn{3}{|l|}{ Histologic grade } \\
\hline G1 & 25 & 9.3 \\
\hline G2 & 107 & 39.9 \\
\hline G3 & 80 & 29.9 \\
\hline Unknown & 56 & 20.9 \\
\hline \multicolumn{3}{|c|}{ Oestrogen receptor status (ER) } \\
\hline Postive & 147 & 54.9 \\
\hline Negative & 104 & 38.8 \\
\hline Unknown & 17 & 6.3 \\
\hline \multicolumn{3}{|l|}{ Types of surgery } \\
\hline $\mathrm{BCS}$ & 72 & 26.9 \\
\hline Mastectomy & 196 & 73.1 \\
\hline
\end{tabular}

as the time from the date of relapse to death of any cause. Analysis of the overall survival according to site of distant relapse was carried out on patients with only one site of distant relapse. Univariate and multivariate analyses were used to analyse demographics and clinicopathological factors. Overall survival was analysed using the Kaplan and Meier method and compared by the log rank test.

\section{Results}

\section{Patient Demographics}

A total of 268 patients diagnosed with non-metastatic invasive breast cancer were identified. The mean age was 50 (range 26-81). Chinese ethnic group scored the highest incidence rate followed by Malay and Indian. The most common tumour size was between 2 and $5 \mathrm{~cm}$. Half of the population had positive axillary lymph node involvement at diagnosis. The most prevalent tumour grade was grade 2 followed by grade 3 and grade 1 . More than $50 \%$ of the patients were ER positive. Majority of patients underwent mastectomy (73.1\%) while the remainders had breast conservation surgery (BCS) (Table 1).

Following BCS, only $81.9 \%$ had adjuvant radiotherapy (RT) to the conserved breast whereas half of the patients who underwent mastectomy received adjuvant RT. Most patients received adjuvant systemic therapy which included either chemotherapy or Tamoxifen or both (Table 2).

Table 2: Types of adjuvant therapy

\begin{tabular}{lll}
\hline \multirow{2}{*}{ Adjuvant treatment } & \multicolumn{2}{c}{ Types of surgery } \\
\cline { 2 - 3 } & $\begin{array}{l}\text { BCS } \\
\mathbf{n}=\mathbf{7 2 ( \% )}\end{array}$ & $\begin{array}{l}\text { Mastectomy } \\
\mathbf{n}=\mathbf{1 9 6}(\%)\end{array}$ \\
\hline Radiotherapy & $59(81.9)$ & $132(67.3)$ \\
Systemic therapy ${ }^{\#}$ & $65(90.3)$ & $186(94.9)$ \\
\hline
\end{tabular}

Note: Some patients received both radiotherapy and systemic therapy for adjuvant treatment, hence the total percentages are greater than $100 \%$.

"Includes chemotherapy and tamoxifen

\section{Patterns of relapse}

At a median follow up of 50 months (range 5 -107 months), 73 patients (27.2\%) developed 
relapse. A total of 15 patients (5.5\%) had local relapse, 5 patients $(1.9 \%)$ had regional relapse and 53 patients $(19.8 \%)$ had distant relapse. Within the distant relapse category, 22 patients (41.5\%) had more than one site of relapse and lung was the most common site of distant relapse $(47.2 \%)$ (Table 3 and 4).

The median DFI was 29 months (range 1-92 months). The relapse rate was $3.7 \%$ at 12 months after surgery, peaked to $9.7 \%$ at 24 months and later declined to $2.4 \%$ at 36 months. The risk of relapse declined steadily after five years to less than $5.0 \%$, as shown in Figure 1.

\section{Clinicopathological factors associated with relapse}

Logistic regression analyses showed that stage, axillary node and oestrogen receptor status had statistically significant correlation with relapse, with $p=0.001$, $p=0.001$ and $p=0.042$, respectively. The data are illustrated in Table 5 below (Table 5).
Table 3: Relapse rate

\begin{tabular}{llrr}
\hline Sites of relapse & $\mathbf{n}=\mathbf{2 6 8}$ & \multicolumn{2}{c}{$\%$} \\
\hline Not relapse & 195 & & 72.8 \\
Relapsed & 73 & & 27.2 \\
$\quad$ Local relapse & 15 & & 5.5 \\
$\quad$ Regional relapse & 5 & 1.9 \\
$\quad$ Distant relapse & 53 & & 19.8 \\
\hline
\end{tabular}

Table 4: Number of relapse according to sites of distant relapse

\begin{tabular}{lcc}
\hline Sites of distant relapse & $\begin{array}{c}\text { Number of } \\
\text { patients }\end{array}$ & $\mathbf{\%}$ \\
\hline Bone & 24 & 45.3 \\
Lung & 25 & 47.2 \\
Liver & 8 & 15.1 \\
Brain & 9 & 16.9 \\
Others & 9 & 16.9 \\
\hline
\end{tabular}

Note: Patients may have more than one site of distant relapse, hence the total percentage is greater than $100 \%$.

*Other sites involved were ovary, peritoneum and adrenal gland

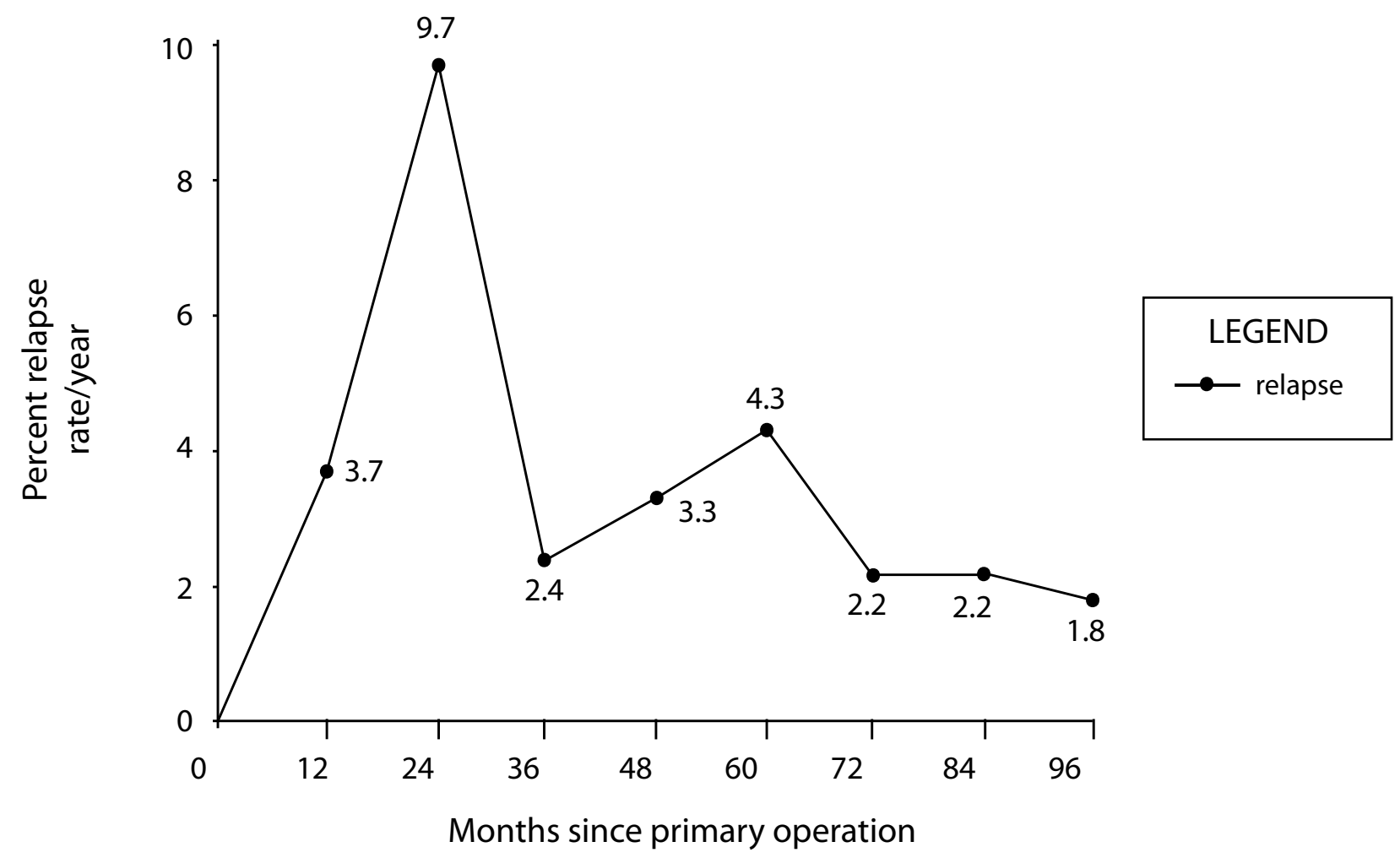

Figure 1: Yearly risk of relapse after primary surgery for breast cancer 


\section{Overall Survival}

\section{Overall survival according to disease free interval}

More than half of the relapses (56.2\%) occurred within the first 36 months after primary surgery. This duration was subsequently chosen to differentiate between short (less than 36 months) or long (more than 36 months) DFI in this study population. Short DFI resulted in lower overall survival rate than long DFI $(p<0.001)$ (Figure 2).

\section{Overall survival according to sites of relapse}

Patients with only local relapse had the best prognosis with a 5-year overall survival of $61 \%$ compared to patients who developed regional or distant relapse with $40 \%$ and $21 \% 5$-year overall survival rate respectively $(p<0.01)$ (Figure3).

\section{Overall survival according to sites of distant relapse}

Two-year overall survival rate for patients with bone metastasis only was $61 \%$ compared to $20 \%$ in those with lung metastasis. None of the patients with liver or brain metastasis were alive at 2 years while patients with relapse at other sites had 2-year overall survival of $50 \%$ (Figure 4).

\section{Discussion}

This study involved 268 patients with the mean age of 50 years (range 26-81 years). The highest incidence was in the Chinese ethnic group and this is consistent with the Malaysian NCR 2003-2005 report (1).

Approximately $18.1 \%$ of patients who had BCS in this study population did not receive adjuvant RT, either due to patients' refusal or reasons unknown. RT to the residual breast is a standard indication after a BCS to reduce the risk of relapse in the conserved breast. Two thirds of the patients who had mastectomy received RT. Of particular note was the high proportion of systemic treatment given following the BCS $(90.3 \%)$ or mastectomy $(94.9 \%)$. These figures are higher than as compared to the study done by Elder et al. which reported $62.7 \%$ of BCS and $70.7 \%$ of mastectomy patients receiving systemic treatment (3). This may be explained by the larger proportion of

Table 5: Number of relapse according to clinicopathological factors of primary tumour

\begin{tabular}{|c|c|c|c|}
\hline Characteristics & $\begin{array}{c}\text { Total no. of patients }(X) \\
n=268\end{array}$ & $\begin{array}{l}\text { No. of patients with relapse }(Y) \\
\qquad n=73\end{array}$ & $\begin{array}{l}\text { Percentage } \\
\mathrm{Y} / \mathrm{X} \times 100 \%\end{array}$ \\
\hline \multicolumn{4}{|l|}{ Stage } \\
\hline 1 & 69 & 12 & 17.4 \\
\hline 2 & 121 & 27 & 22.3 \\
\hline 3 & 78 & 34 & 43.6 \\
\hline \multicolumn{4}{|l|}{ Tumour size } \\
\hline$\leq 2 \mathrm{~cm}$ & 95 & 20 & 21.1 \\
\hline$>2$ and $\leq 5 \mathrm{~cm}$ & 139 & 39 & 28.1 \\
\hline$>5 \mathrm{~cm}$ & 33 & 14 & 42.4 \\
\hline Unknown & 1 & 0 & 0.0 \\
\hline \multicolumn{4}{|c|}{ Axillary node status } \\
\hline Node positive & 134 & 48 & 35.8 \\
\hline Node negative & 134 & 25 & 18.7 \\
\hline \multicolumn{4}{|l|}{ Tumour grade } \\
\hline Grade 1 & 25 & 5 & 20.0 \\
\hline Grade 2 & 107 & 30 & 28.0 \\
\hline Grade 3 & 80 & 30 & 37.5 \\
\hline Unknown & 56 & 8 & 14.3 \\
\hline \multicolumn{4}{|c|}{ Oestrogen receptor status } \\
\hline Positive & 147 & 34 & 23.1 \\
\hline Negative & 104 & 37 & 35.6 \\
\hline Unknown & 17 & 2 & 11.8 \\
\hline
\end{tabular}




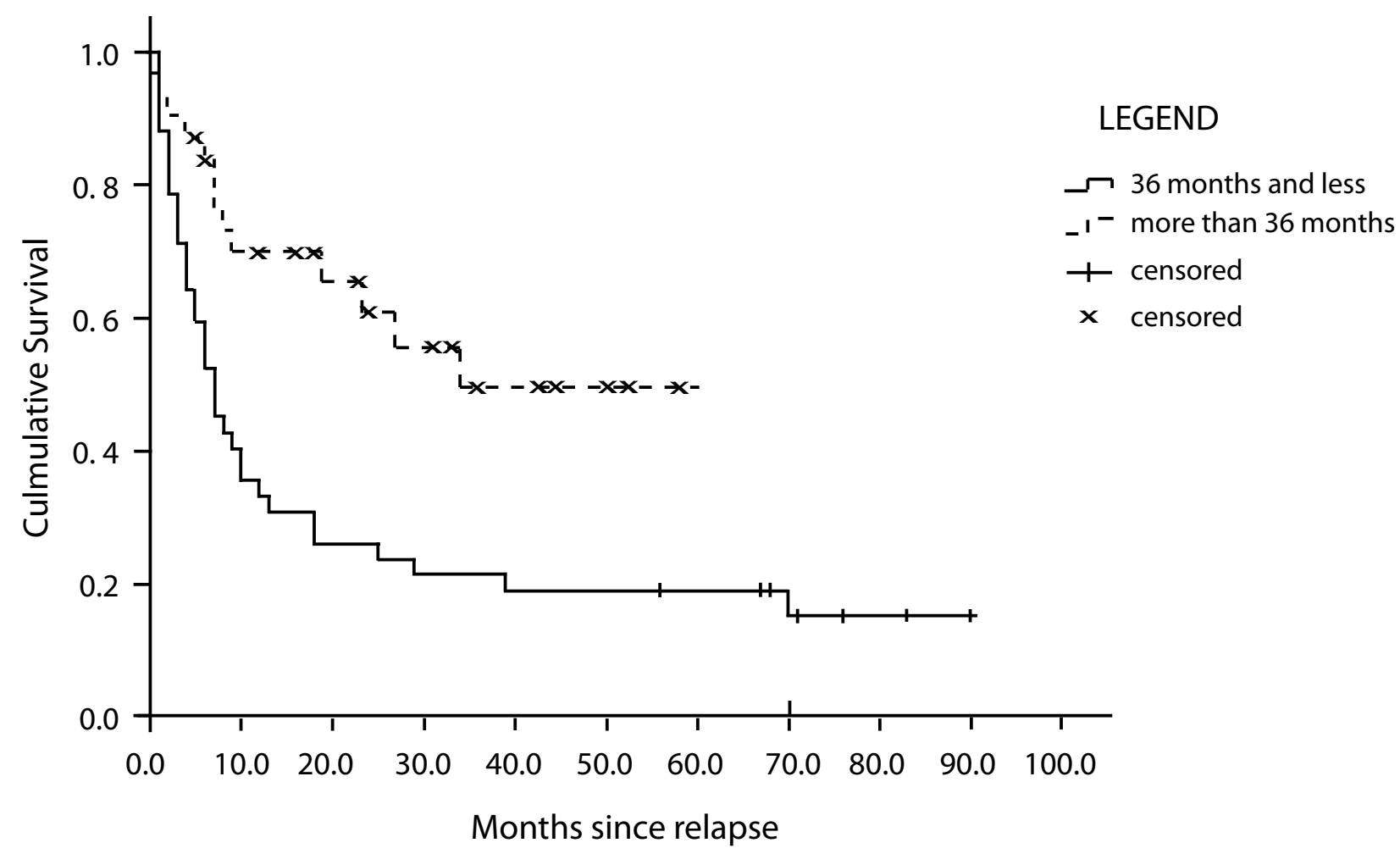

Figure 2: Overall survival according to disease free interval

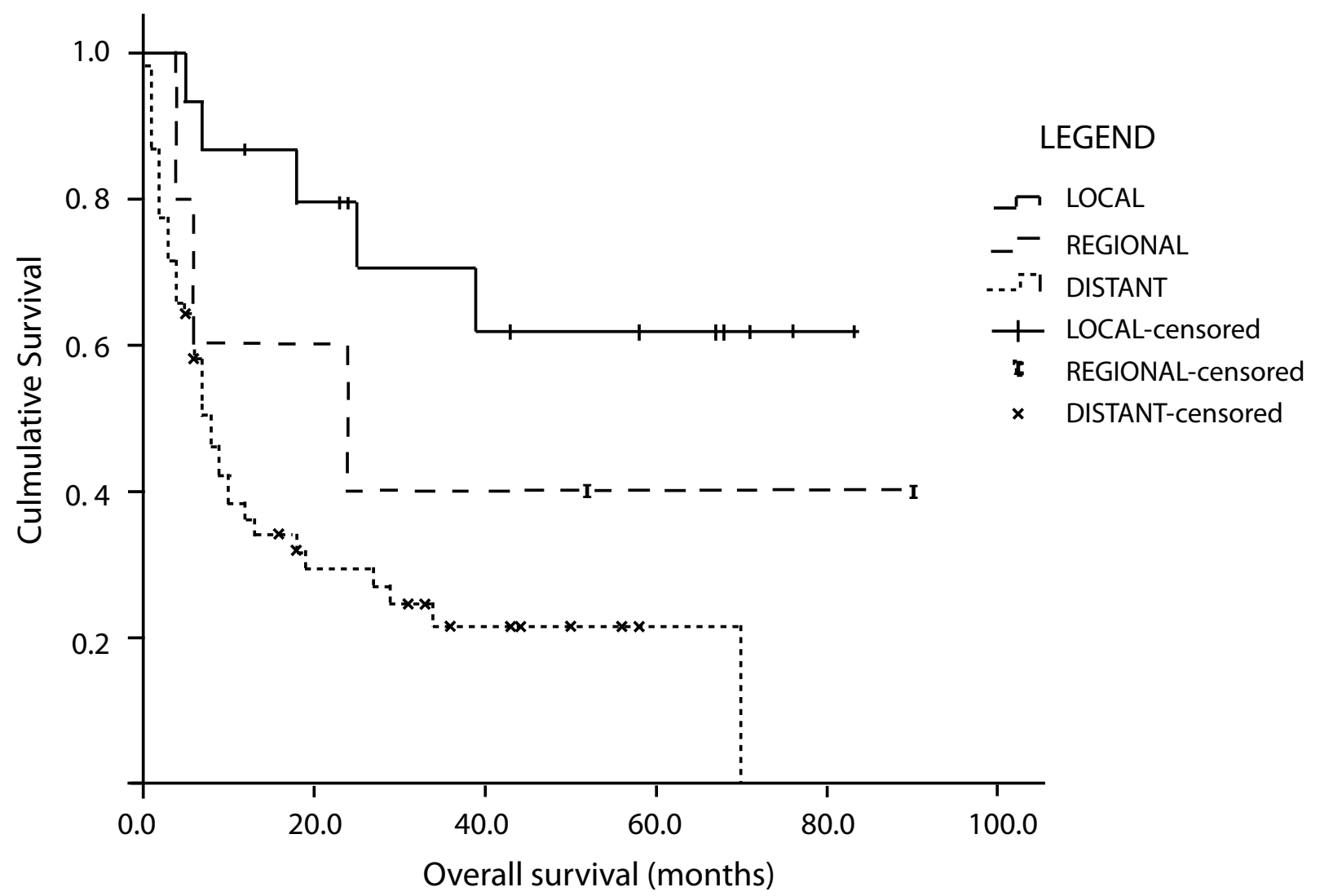

Figure 3: Overall survival according to sites of relapse 


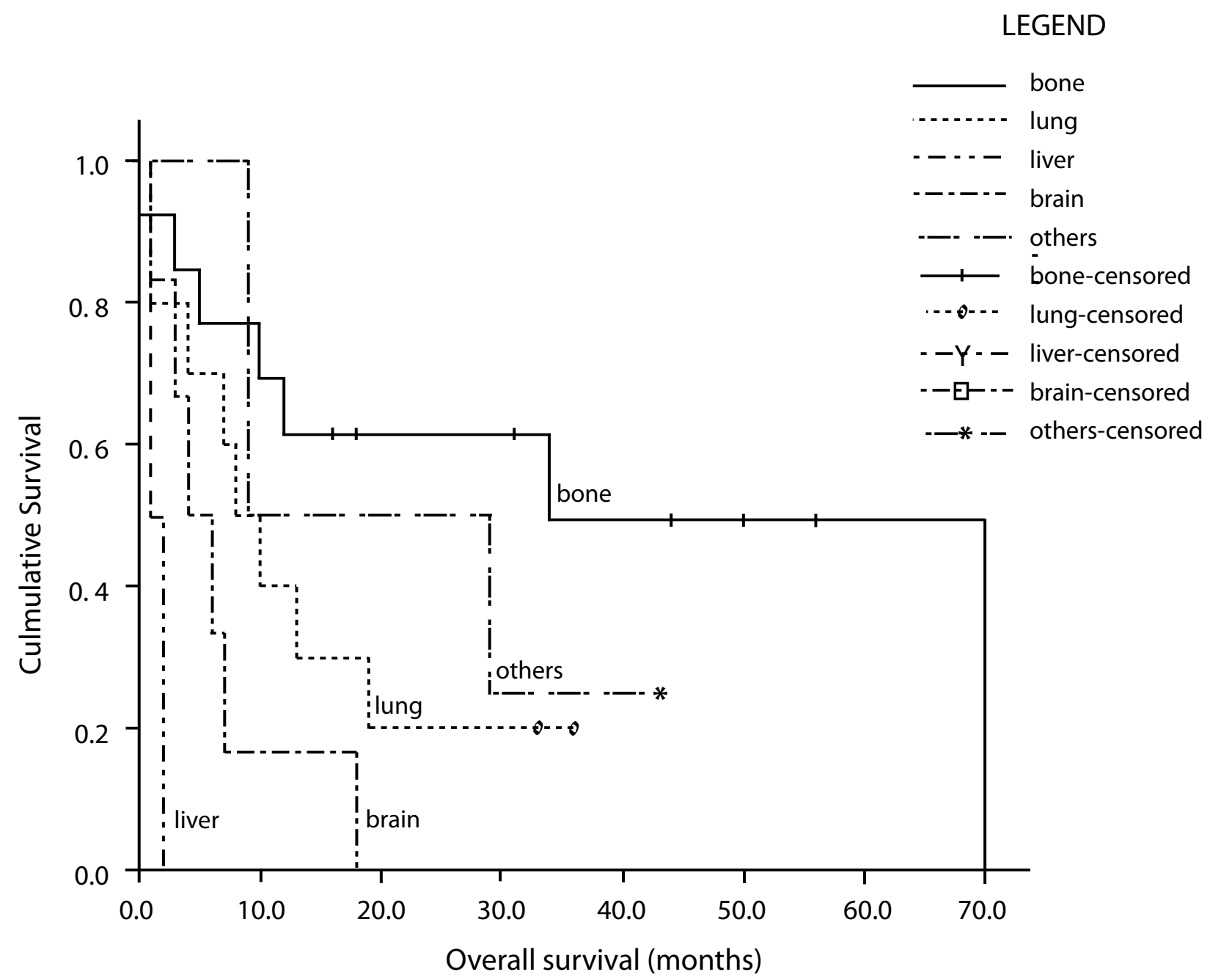

Figure 4: Overall survival according to sites distant relapse.

Note: Analysis was done on patients with only one site of distant relapse.

patients with higher stage disease in this study who require adjuvant systemic treatment. Furthermore, more than half of the study population had ER positive disease and therefore received tamoxifen as part of the systemic treatment.

The relapse rate in this study was $27.2 \%$ with local, regional and distant relapse rates of $5.5 \%$, $1.9 \%$ and $19.8 \%$ respectively. Elder et al. reported a lower relapse rate of $18 \%$ with local, regional and distant relapse rates of $4.9 \%, 1.0 \%$ and $11.4 \%$ respectively (3). The higher relapse rate seen in our patients may be explained by more patients with higher stage disease who were at a higher risk of developing relapse. Stage 3 disease in this study was $29.0 \%$ compared to $3.9 \%$ from the study by
Elder et al (3). The most common site of distant relapse was the lung which accounted for $47.2 \%$ of cases followed by bone, liver, brain and other sites.

Majority of relapse occurred within the first five years from primary surgery, in agreement with published data (3). The highest risk of relapse was between one to two years from surgery $(9.7 \%)$ and beyond five years, the risk of relapse decreased steadily. The median DFI in this study was 29 months. If patients have not relapsed within the first five years of surgery, the likelihood of relapsing thereafter is very small. However, patients with a history of breast cancer suffer excess mortality for more than 30 years after surgical therapy (9). 
Similar to the findings by Elder et al. and Imkampe et al $(3,5)$, tumour stage, axillary node involvement and oestrogen receptor status were found to have statistically significant correlation with relapse in this study. Patients with higher tumour stage, positive nodes and oestrogen receptor positive status were more likely to relapse. However, the correlation between tumour grade and relapse was not significant, $(p=0.07)$. This could be due to high proportion of unknown grade $(14.3 \%)$ in the relapsed cases in this study population and the reasons for this was unclear.

DFI was an important prognostic factor for breast cancer relapse. Patients who had relapse after 36 months had better survival rate than those who relapsed earlier. The difference in survival was statistically significant $(p<0.001)$ and consistent with other studies (3).

Patients with local relapse had better prognosis compared to regional and distant relapse. The fiveyear overall survival for patients with local, regional and distant relapse were $61 \%, 40 \%$ and $21 \%$, respectively. The overall survival was higher compared to the study by Elder et al. which quoted $41 \%$ for local relapse, $20 \%$ for regional and $13 \%$ for distant relapse (3). There were 11 patients (15.1\%) who did not come for follow-up and were not contactable. Therefore, they were deemed as 'unknown'. This could be one of the reasons for the overall higher survival rate seen in this study. Another reason could be due to effective treatment delivered to patients with relapse but this needs further studies to confirm. For those with local relapse, about $66 \%$ had salvage surgery and they were still alive until the end of the study. This may explain the relatively good overall survival for local relapse.

Bone metastases had better prognosis than visceral metastases $(3,5)$. In this study, two-year overall survival for bone metastases was $61 \%$, lung metastases was $20 \%$ and $0 \%$ for those with liver and brain metastases. The reasons for the relatively better prognosis of bone metastases are still unclear. A randomized study looking at the clinical course of bone metastases for breast cancer reported that bone metastases were more common in receptor positive or well differentiated tumours which are associated with better prognosis (10).

\section{Limitations and Recommendations}

This was a retrospective study, therefore, the documentation in the case notes were not standardised and some data were missing or incomplete. The study sample was relatively small compared to other studies.

To ensure more reliable and accurate data, a prospective study with larger sample size, longer duration and a longer follow-up is recommended. A mechanism which allows a continuous data collection through a prospective database, either at an international or a national level, would be beneficial.

\section{Conclusions}

Most relapses occur within the first five years of diagnosis and patients with late relapse have better survival than early relapse. Survival of patients with relapsed breast cancer is associated with the site of first relapse. The prognosis is better for local and regional relapse compared to distant relapse. Clinicopathological factors are useful to predict risk of relapse in patients with breast cancer after completing treatment.

Disease relapse is one of the worries faced by patients with breast cancer after completing their curative therapy. Data on patterns of relapse and its prognosis is both important and useful to clinicians when discussing long term outcome with the patients. This is the first study to provide data on breast cancer relapse in Malaysia. The results will be an invaluable information for our clinicians in the management and counselling of patients with breast cancer.

\section{Acknowledgements}

I would like to thank Professor Yip Cheng Har and Associate Professor Nur Aishah Mohd Taib from the Department of Surgery, Faculty of Medicine, University Malaya Medical Centre (UMMC), for their help in providing the UMMC Breast Cancer Prospective Database for this study.

\section{References}

1. Lim GCC, Halimah Y, Rampal S. Cancer Incidence in Peninsular Malaysia, 2003-2005. The Third Report of the National Cancer Registry, Malaysia. 
2. Parkin MD, Bray F, Ferlay J, Pisani P. 2005, 'Global Cancer Statistics, 2002', CA Cancer J Clin; 55: 74108.

3. Elder $\mathrm{EE}$, et al. Patterns of breast cancer relapse. EJSO 2006; 32: 922-927.

4. MOH Clinical Practice Guidelines of 'Management of Breast Cancer', Dec 2002.

5. Imkampe A, et al. The significant of the site of recurrence to subsequent breast cancer survival. EJSO 2007; 33: 420-423.

6. Giordano $\mathrm{SH}$, et al. Is breast cancer surviving improving? Cancer 2004; 100: 44-52.

7. Taib NA, Yip CH, Ibrahim M. Survival Analyses of 413 Malaysian Women with Breast Cancer: Results from the University Malaya Medical Centre. Asian Pac J Cancer Prev 2008; 9(2): 197-202.
8. Hess KR, et al. Estrogen receptors and distinct patterns of breast cancer relapse. Breast Cancer Res Treat 2003 Mar; 78(1):105-118.

9. Hibberd AD, Horwood LJ, Wells JE. Long term prognosis of women with breast cancer in New Zealand: study of survival to 30 years. $\mathrm{Br}$ Med J (Clin Res Ed) 1983; 286: 1777-1779.

10. Coleman RE, Rubens RD. The clinical course of bone metastases from breast cancer. Br J Cancer 1987; 55: 61-66. 